\title{
SAT, SMT and Applications
}

\author{
Armin Biere \\ Johannes Kepler University, Linz, Austria
}

\begin{abstract}
SAT solving has gained tremendous interest. On the practical side there have been considerable performance improvements, due to new highly efficient algorithms, new heuristics, and optimized data structures. There are new applications and reformulations of important classical problems, mainly in the context of formal methods, where SAT solving is also applied successfully in an industrial setting. These applications range from equivalence checking, configuration, over model checking to test case generation. SAT is becoming one of the most important core technology in all these areas. Many applications actually use Satisfiability Modulo Theory (SMT), which can be seen as an extension of SAT solving. SMT has it roots in automated theorem proving. But it heavily relies on SAT technology. We discuss some key technologies in practical SAT solving, e.g. how to write a fast solver, some aspects in lifting propositional SAT technology to richer domains, how competitions can help to improve the state-of-the-art and finally touch on applications in model checking, hardware and software verification.
\end{abstract}

\section{References}

1. Biere, A.: PicoSAT essentials. Journal on Satisfiability, Boolean Modeling and Computation (JSAT) 4 (2008)

2. Biere, A.: Tutorial on model checking: Modelling and verification in computer science. In: Horimoto, K., et al. (eds.) AB 2008. LNCS, vol. 5147, pp. 16-21. Springer, Heidelberg (2008)

3. Biere, A., Heule, M., van Maaren, H., Walsh, T. (eds.): Handbook of Satisfiability. IOS Press, Amsterdam (2009)

4. Prasad, M., Biere, A., Gupta, A.: A survey on recent advances in SAT-based formal verification. Software Tools for Technology Transfer (STTT) 7(2) (2005) 